\title{
Plasma infusions in thrombotic thrombocytopenic purpura complicating systemic lupus erythematosus-a successful outcome
}

\author{
R. FINKELSTEIN \\ M.D. \\ A. MARKEL
M.D.
}

\author{
A. CARTER \\ M.D. \\ J. G. BROOK \\ M.R.C.P.
}

Departments of Internal Medicine " $B$ " and Hematology, Rambam Medical Center, Technion-Faculty of Medicine, Haifa, Israel

\section{Summary}

A severe form of acute thrombotic thrombocytopenic purpura (TTP) developed in a patient with systemic lupus erythematosus (SLE). Infusions of large amounts of fresh frozen plasma (FFP) were added to steroid therapy and resulted in a rapid improvement and remission. Further episodes of thrombocytopenia and abdominal pains during a two-year follow-up were successfully treated with plasma alone and this indicates the important role of FFP infusions in the recovery of this patient.

\section{Introduction}

Thrombotic thrombocytopenic purpura (TTP) is characterized clinically by thrombocytopenia, microangiopathic haemolytic anaemia, fever, fluctuating neurologic signs and renal dysfunction. Histology shows diffuse thrombotic occlusions in which platelets and fibrin are found (Amorosi and Ultmann, 1966). The complexity of these clinical findings have led to speculation that TTP is a syndrome of diverse aetiologies (Umlas and Kaiser, 1970). The outcome is almost invariably fatal and since pathogenesis remains ill-understood, many therapeutic regimens have been employed with undefined success. Among these, five are particularly advocated. Splenectomy (Bernard, Bonman and Schwartz, 1969), antiplatelet agents (Amir and Kraus, 1973), high doses of glucocorticoids (Burke and Hartman, 1959), exchange transfusions (Bukowski et al., 1976) and plasmapheresis (Bukowski, King and Hewlett, 1977). More recently Byrnes and Khurana (1977) successfully treated a patient with TTP with plasma transfusions.

We describe a patient with an overlapping clinical picture of systemic lupus erythematosus (SLE) and TTP whose response to high doses of glucocorticoids was equivocal but who showed a dramatic improvement and remission after repeated plasma infusions.

\section{Case report}

A 27-year-old woman with a one-year history of weakness, arthralgia and alopecia was admitted to another hospital because of lymphadenopathy, anaemia and thrombocytopenia. On examination she was pale, with diffuse lymphadenopathy and palpable spleen; the right leg was the site of a large ecchymosis. The neurological examination was normal. The haemoglobin was $6.0 \mathrm{~g} / \mathrm{dl}$, the haematocrit $20 \%$, the reticulocyte count $4.8 \%$, the white cell count $5.7 \times 10^{9} / 1$ and the platelet count was $48 \times 10^{9} / 1$. No microangiopathic changes were apparent on the peripheral blood smear. An LE cell preparation and an antinuclear antibody test were positive. The plasma urea and the creatinine were normal and no relevant findings were found on urine examination.

Four days after admission profuse vaginal bleeding developed, the platelet count decreased to $20 \times 10^{9} / 1$ and the haemoglobin to $3.9 \mathrm{~g} / \mathrm{dl}$. The patient suffered headache, abdominal pain and a fever of $38.5^{\circ} \mathrm{C}$. Five days later the platelet count was $6 \times 10^{9} / 1$. The patient became lethargic, confused and developed a left sided hemiparesis. Glucocorticoids were instituted at a dose of $200 \mathrm{mg}$ of prednisolone daily. She was then transferred to our hospital.

Fundus examination revealed numerous small haemorrhages without exudates or papilloedema. A computed tomographic study (CT scan) of the brain was normal. The haemoglobin was $9.5 \mathrm{~g} / \mathrm{dl}$, the haematocrit $29.4 \%$, the white cell count $6.4 \times 10^{9} / 1$, the reticulocyte count $31 \cdot 1 \%$ and the platelet count $18 \times 10^{9} / 1$. The peripheral blood smear showed marked red blood cell fragmentation and multiple nucleated red blood cells, compatible with a micro- 
angiopathic type of anaemia. The plasma urea was $10.71 \mathrm{mmol} / \mathrm{l}$, and the creatinine $106 \mu \mathrm{mol} / \mathrm{l}$. Urinalysis revealed many red blood cells and there was a proteinuria of $1.5 \mathrm{~g} / 24 \mathrm{hr}$; the total bilirubin was 37.6 $\mu \mathrm{mol} / 1$ (10.2 $\mu \mathrm{mol} / 1$ conjugated). The serum lactic dehydrogenase was $11 \mu \mathrm{mol} / \mathrm{sec} / 1$ and haptoglobin was not present in the serum. The direct Coombs' test was negative, and the serum fibrinogen, fibrinogen split products, prothrombin time, partial thromboplastin time and thrombin time were all within normal limits. Double stranded anti-DNA antibodies were present and the serum $C_{3}$ and $C_{4}$ were both below the lower limits of the normal. A gingival biopsy was normal.

Treatment with the same dose of prednisolone was continued but, on the basis of a clinical diagnosis of TTP, transfusions of fresh frozen plasma were begun. The patient's haematological values during this therapy are detailed in Fig. 1 . Between the 2 nd and 5 th day of admission she received a total of 37 units of fresh frozen plasma (FFP) and 17 units of thrombocytes. There was a marked improvement of her general condition within hours of the first FFP transfusion and a gradual but complete recovery of the neurological findings took place over the next four days. The platelet count increased to $282 \times 10^{9} / 1$. Over the next 10 days no further plasma was administered and the prednisolone dose was decreased to $40 \mathrm{mg}$ daily. During this period the patient complained of severe abdominal pains and when the platelet count dropped suddenly to $59 \times 10^{9} / 1$, plasma infusions were reinstituted. Thrombocytes were not transfused on this occasion. The dose of prednisolone was increased to $100 \mathrm{mg} /$ day over the next 2 days $c$ and then rapidly diminished for the following 5 days. The benefit was immediate; the pain ceased, the $\stackrel{5}{+}$ platelet count increased to $290 \times 10^{9} / 1$ and remained $\frac{}{c}$ such despite the rapid reduction in the steroid dosage.

By the time of discharge, all manifestations of TTP $\frac{\bar{\sigma}}{\widehat{D}}$ and its complications had resolved and the patient $\stackrel{\odot}{\mathscr{Q}}$ was in a sustained remission. She had received a total of 50 units of FFP.

The patient has been followed as an out-patient for a period of two years. On three occasions she has developed bouts of abdominal pain with a drop in the thrombocyte count. During these episodes she received plasma transfusions on each occasion and 3 improved. Now, two years subsequent to discharge she remains well, free of complaints and with a normal haematological picture.

\section{Discussion}

Despite the lack of histological evidence, our patient exhibited all the characteristic clinical features of TTP. Furthermore, there were also ample clinical grounds for a diagnosis of SLE.

Considerable overlap between SLE and TTP on both clinical (Amorosi et al., 1966) and anatomica grounds (Levine and Shearn, 1964) is known to exist In a review of 151 cases of TTP (Levine and Shearn, 1964) the additional presumptive diagnosis of SLE was made in 34 of these patients.

The pathogenesis of TTP is still unknown. The

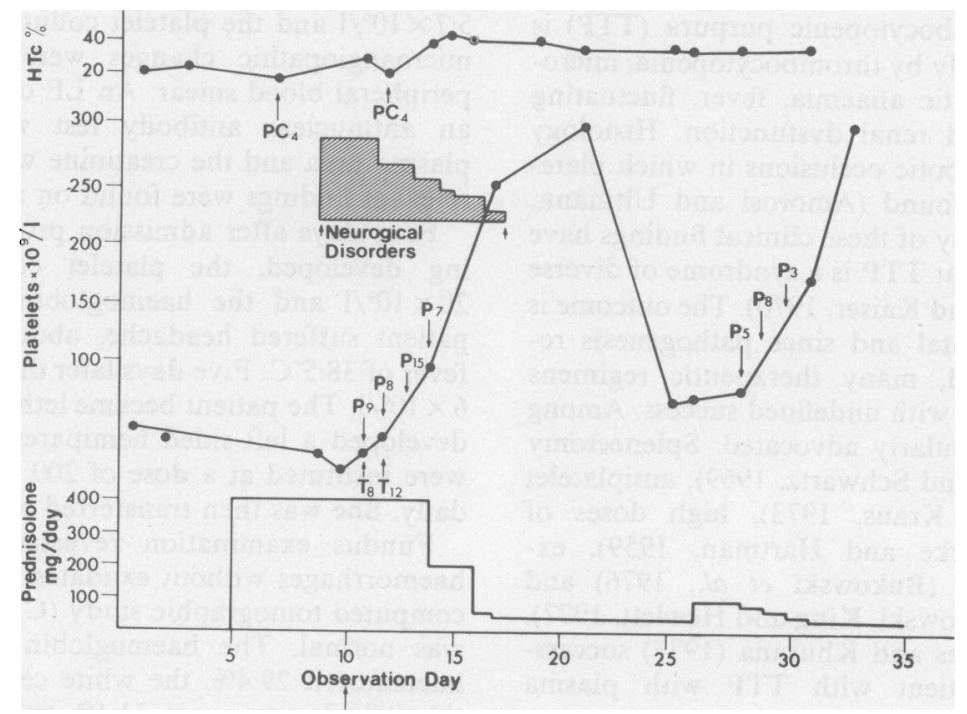

FIG. 1. Clinical course through the 35th day of observation as manifested by the platelet count, haematocrit (HTc) and neurological status. 'PC' indicates transfusion of packed red blood cells, ' $P$ '-infusion of fresh frozen plasma and ' $T$ ' $\rightarrow$ thrombocyte transfusions. The number appearing beside the letters 'P', 'PC' and ' $T$ ' represents the number of units of transfusions administered. Further details are provided in the text. 
treatment therefore has generally been empirical or with an assumed rationale. The use of plasma infusion is based on observations that exchange transfusion and plasmapheresis have resulted in a remarkable improvement and remission in many patients with TTP (Bukowski et al., 1976, 1977). The mechanism for the effectiveness of these procedures was thought to be related to removal of an initiating or damaging agent, possibly immune complexes. However, the observation that the disorder can be successfully treated by simple plasma infusions raised new questions concerning the pathogenesis and treatment of this disease. Until now, we are aware of 12 other patients with TTP that have been treated by plasma infusion and in 10 of them the treatment was highly effective (Byrnes and Lian, 1979). In three of these patients, plasma collected during relapse induced, in vitro, aggregation of washed platelets from both normal donors and patients during remission. It was thus postulated that TTP appears to be due to a deficiency of a plasma inhibitor that counteracts a platelet aggregating factor present in normal and TTP plasma. In another patient, the disease was exacerbated during plasmapheresis when albumin-saline was used for volume replacement, and the manifestations remitted with subsequent whole blood exchange transfusions (Reiss et al., 1978).

Whatever the exact mechanism, it is apparent that TTP is most frequently responsive to replacement of a plasma factor and the effectiveness of exchange transfusion and plasmapheresis is due to replaced plasma and not the removal of an unknown factor, toxin or immune complexes from the circulation (Byrnes and Lian, 1979).

The clinical course and the rapid improvement observed in our patient is in accord with similar cases where plasma infusions were successfully used. Although it is impossible to state that she failed to respond to the high dose of corticosteroids it appeared that the plasma infusions resulted in the dramatic change in the clinical course of the patient. The post-hospitalization occurrence of several at- tacks of abdominal pain associated with thrombocytopenia which responded to plasma infusions alone gives added credence to our assumption regarding the therapeutic efficacy of this mode of treatment in this patient. Nalbadian, Henry and Rich (1979) have stated that plasma infusion is the most important therapeutic innovation in the history of TTP. To date, insufficient experience with this form of treatment exists for specific guidelines for the use of plasma to be established. There is no doubt, however, that this therapeutic approach raises the most hope for the very ill patient with TTP.

\section{References}

AMOROSI, E.L. \& ULTMANN, J.E. (1966) Thrombotic thrombocytopenic purpura: Report of 16 cases and review of literature. Medicine (Baltimore), 45, 139.

AMIR, J. \& KRAUS, S. (1973) Treatment of thrombocytopenic purpura with antiplatelet drugs. Blood, 42, 27.

BERNARD, R.P., BoNMAN, S.L. \& SCHWARTZ, S.L. (1969) Splenectomy for thrombotic thrombocytopenic purpura. Annals of Surgery, 169, 616.

BURKE, H.A. \& HARTMAN, R.C. (1959) Two patients with remission associated with the use of large amounts of steroids. Archives of Internal Medicine, 103, 105.

Bukowski, R.M., HewletT, J.S., HaRRIS, J.W., HoffMaN, G.C., BATTLE, J.R., SilverblatT, E. \& YANG, I.Y. (1976) Exchange transfusions in the treatment of thrombotic thrombocytopenic purpura. Seminars in Hematology, 13, 219.

BuKowski, R.M., KING, J.W. \& HeWLETT, J.S. (1977) Plasmapheresis in the treatment of thrombotic thrombocytopenic purpura. Blood, 50, 413

BYRNES, J.J. \& KhuRANA, M. (1977) Treatment of thrombotic thrombocytopenic purpura with plasma. New England Journal of Medicine, 297, 1386.

BYRNES, J.J. \& LIAN, E.C.Y. (1979) Recent therapeutic advances in thrombotic thrombocytopenic purpura. Seminars in Thrombosis and Hemostasis, 5, 199.

LEVINE, S. \& SHEARN, M. (1964) Thrombotic thrombocytopenic purpura and systemic lupus erythematosus. Archives of Internal Medicine, 113, 826.

Nalbadian, R.M., HenRY, R.L. \& BiCH, R.L. (1979) Thrombotic thrombocytopenic purpura. An extended editorial. Seminars in Thrombosis and Hemostasis, 5, 216.

Reiss, R., ShaH, V., Kalter, R. \& Panlilio, A. (1978) Plasmapheresis in thrombotic thrombocytopenic purpura (TTP). (Letter). Blood, 51, 559.

UMLAS, J. \& KAISER, J. (1970) Thrombotic thrombocytopenic purpura: a disease or a syndrome? American Journal of Medicine. 49, 723. 